\title{
Entscheidungsverhalten von Paaren in materiell prekären Lagen über größere Anschaffungen und die Freizeitgestaltung
}

\section{Decision making within couples in deprived circumstances concerning major purchases and leisure time activities}

\begin{abstract}
Zusammenfassung:
Der vorliegende Artikel analysiert die Aussagen von Paaren zur Verteilung der Entscheidungsmacht innerhalb der Partnerschaft. Mit den Daten der zweiten Welle der Panelbefragung ,Arbeitsmarkt und soziale Sicherung" wird insbesondere die Machtverteilung bei Paaren in materiell prekären Lagen untersucht, die wir über den Arbeitslosengeld-II-Bezug (ALG II, ugs. „Hartz IV“) definieren. Über größere Anschaffungen entscheidet in prekären Lagen eher die Frau alleine. Die Freizeitgestaltung wird dagegen bei diesen Paaren seltener alleine von der Frau bestimmt. In multinomialen, logistischen Regressionsmodellen zeigt sich, dass für die Verteilung der Entscheidungsmacht bei den ALG-II-Paaren z.T. andere Einflussfaktoren maßgeblich sind als bei Paaren ohne Bezug. Die ökonomischen Ressourcen, die die beiden Partner in die Partnerschaft einbringen, haben im Wesentlichen nur für Paare ohne ALG-II-Bezug Bedeutung. Eine modernere Geschlechterrolleinstellung ist unseren Analysen nach in beiden Gruppen häufiger mit einem egalitären Entscheidungsverhalten bzgl. größerer Anschaffungen verknüpft. Bei Entscheidungen über die Freizeitgestaltung ist dieser Einfluss wiederum nur bei den Nicht-Beziehern zu finden. Daneben zeigen sich weitere Faktoren, die die Verteilung der Entscheidungsmacht beeinflussen. Auffällig ist z.B. das egalitärere Entscheidungsverhalten von Personen mit niedriger Bildung im ALG-II-Bezug.
\end{abstract}

Schlagwörter: Entscheidungsmacht, Partnerschaft, ALG II

\begin{abstract}
:
This article analyses the division of decision power within couples. Based on data from the second wave of the panel study "Labour Market and Social Security", the allocation of power between the spouses is studied with special regard to couples receiving the German „Unemployment Benefit II". In deprived circumstances, decisions on major purchases are more often made solely by the woman. On the other hand, decisions on leisure time activities among these couples are less often taken by the women alone. Multinomial logistic regressions indicate that the distribution of decision power among couples in receipt of benefits is governed by other factors than among couples without receipt. The influence of the economic resources that both partners contribute is only visible in the non-recipient group. In both groups, modern gender role attitudes are often connected with a more egalitarian decision-making process regarding major purchases. Again for decisions about leisure time activities, this relation can only be found among the non-recipients. Apart from these, we find additional factors influencing the division of decision power, like individuals with low education deciding more egalitarian in the Unemployment Benefit II group.
\end{abstract}

Key words: decision power, couples, welfare benefits 


\section{Einleitung}

Ein in sozialwissenschaftlichen Studien zu Ungleichheiten zwischen den Geschlechtern häufig diskutierter Aspekt sind Machtungleichheiten zwischen den Partnern. In diesem Zusammenhang ist häufig die Kontrolle über das Geld in der Partnerschaft oder die Kontoführung der zentrale Untersuchungsgegenstand (vgl. Ludwig-Mayerhofer 2000, 2006; Pahl 1980, 1983, 1990, 1995; Vogler 1998; Vogler/Lyonette/Wiggins 2008; Vogler/Pahl 1993). Die Prozesse der Entscheidungen des Paares über Anschaffungen (vgl. Kirchler 1995; Kirchler/Hoelzl/Kamleitner 2008; Lise/Seitz 2011) oder auch über Spendenausgaben (vgl. Andreoni/Brown/Rischall 2003; Wiepking/Bekkers 2010) sind ebenfalls Thema zahlreicher Studien.

Ein Teil der Studien analysiert, inwiefern in Niedrigeinkommenshaushalten andere Mechanismen der Machtverteilung - insbesondere mit Blick auf die Allokation des Geldes zwischen den Partnern - wirken, als in finanziell besser gestellten Partnerschaften (vgl. Addo/Sassler 2010; Lott 2009; Ludwig-Mayerhofer/Gartner/Allmendinger 2006; Pahl 1980). Bereits 1980 kommt Pahl (1980) zu dem Befund, dass in Niedrigeinkommenshaushalten im Gegensatz zu besser gestellten Haushalten die Geldverwaltung eher als Belastung denn als Machtinstrument wahrgenommen wird und eher den Frauen zufällt. Nye (1978, 1980) schlussfolgert dementsprechend auch, dass die Verwaltung des Geldes mit Kosten verbunden ist und der Partner über mehr Macht verfügt, der diese abgeben kann und somit autonom ist.

Allerdings sind in diesen Untersuchungen Haushalte mit Erwerbseinkommen Gegenstand der Betrachtung. Im vorliegenden Artikel nähern wir uns dem Zusammenhang von prekärer sozio-ökonomischer Lage und Machtungleichheit innerhalb der Partnerschaft durch die Berücksichtigung des ALG-II-Bezugs mit einer bisher vernachlässigten Schwerpunktsetzung. Neben Haushalten, die noch über ein eigenes Erwerbseinkommen verfügen (den sogenannten „Aufstockern“), beinhaltet die Gruppe der ALG-II-Bezieher eine große Zahl von Personen ohne eigenes Einkommen.

Für Paare stellt der ALG-II-Bezug eine besondere Situation dar. Im Vergleich zu sonstigen Niedrigeinkommensbeziehern ist die gegenseitige Abhängigkeit zwischen den Partnern, die von der staatlichen Grundsicherung (dem Arbeitslosengeld II, umgangssprachlich häufig als „Hartz IV“ bezeichnet) leben, noch stärker ausgeprägt. Der Gesetzgeber geht davon aus, dass Partner, die miteinander verheiratet sind oder in einer ähnlich engen Intimbeziehung innerhalb eines Haushaltes leben, füreinander einstehen müssen und eine sog. „Bedarfsgemeinschaft“ bilden ( $\$ 7$ Absatz 3 SGB II). An dieses Konstrukt ist auch der Bezug von staatlichen Transferleistungen gekoppelt, die in solchen Fällen oft die einzige Einkommensquelle bilden. Die materiellen Unterstützungsleistungen des SGB II sind so bemessen, dass einerseits eine schnelle Wiederaufnahme einer Erwerbstätigkeit angestrebt wird, andererseits jedoch das Existenzminimum im Sinne einer Grundsicherung gewährleistet wird (vgl. Hirseland/Ramos Lobato 2010: 20ff.).

Im Rahmen des SGB II-Grundsatzes des „Förderns und Forderns“ können Versäumnisse und Pflichtverletzungen seitens des Beziehers auch durch Leistungskürzungen sanktioniert werden, wovon schließlich die ganze Bedarfsgemeinschaft betroffen ist (vgl. Götz/Ludwig-Mayerhofer/Schreyer 2010). Die Erwerbslosigkeit eines Partners kann auch dazu führen, dass der andere Partner gezwungen wird, sein Arbeitsangebot auszuweiten, 
nicht nur um das durch die Erwerbslosigkeit verringerte Haushaltseinkommen auszugleichen, sondern auch um institutionalisierten Zwangsmaßnahmen zu entgehen. Dadurch stellt sich die Schicksalsgemeinschaft der beiden Partner noch enger dar als bei Partnerschaften ohne Leistungsbezug.

Folgt man einschlägigen Befunden zur Belastung von Arbeitslosen (vgl. Grün/Hauser/Rhein 2008; Jahoda/Lazarsfeld/Zeisel 2007 [1933]; Mohr/Richter 2008), kann angenommen werden, dass neben den geringen Möglichkeiten materieller Teilhabe auch psychische Belastungsfaktoren die Betroffenen beeinträchtigen, die ebenfalls Rückwirkungen auf das Zusammenleben der Partner haben können. Für ALG-II-Bezieher kommen Wenzel (2008), sowie Hirseland/Ramos Lobato (2010) zu ähnlichen Befunden und benennen als typische Wahrnehmungs- und Verarbeitungsmuster von ALG-II-Beziehern unter anderem Gefühle des Autonomieverlustes wie auch der Exklusion. Nach Lenz (2009: 126) kann Arbeitslosigkeit einen Wendepunkt in Partnerschaftsbeziehungen hinsichtlich der Machtbalance sowie in Bezug auf den Alltag von Beziehungen darstellen. In einer 1991 durchgeführten Studie betonen Hess, Hartenstein und Smid (1991: 186ff.), dass bei Arbeitslosigkeit vor allem die Stellung des Mannes in der Familie durch den Verlust seiner Rolle als Ernährer beeinträchtigt wird und sich durch die ständige Anwesenheit des Mannes Veränderungen für die Beziehung ergeben.

Für Partnerschaften, die ALG-II-Leistungen beziehen, ergibt sich somit eine besonders prekäre Lage, da über das Konzept der Bedarfsgemeinschaft die ökonomische Abhängigkeit zwischen den Partnern noch verstärkt wird. Dieser Artikel untersucht, welche besonderen Auswirkungen dies auf die Machtverteilung in der Partnerschaft haben kann.

Die zitierten Studien beschäftigen sich vornehmlich mit Entscheidungen zu ökonomischen Aspekten in der Partnerschaft. Daneben gibt es aber auch andere bedeutende Bereiche der partnerschaftlichen Lebensführung. Da wir davon ausgehen, dass die Muster und Prozesse der Machtverteilung in anderen Bereichen anders strukturiert sein können, erweitern wir die Analysen auf die Untersuchung der Machtverteilung bei der Gestaltung der gemeinsamen Freizeit.

Bisher liegen kaum Befunde vor, die explizit das Entscheidungsverhalten über die partnerschaftliche Freizeitgestaltung thematisieren. ${ }^{1}$ Studien, die sich mit dem Entscheidungsverhalten von Paaren in diesem Kontext beschäftigen, finden sich hauptsächlich in der Literatur der Reise- und Tourismusforschung. Dabei ist häufig die Entscheidungsmacht der Partner hinsichtlich der gemeinsamen Urlaubsplanung ein Untersuchungsgegenstand (vgl. Cosenza/Davis 1981; Fodness 1992; Mottiar/Quinn 2004; Nichols/Snepenger 1988; van Raaji/Francken 1984).

Wie Fodness (1992: 12) zeigt, sind Frauen häufiger die „family information seeker“, d.h. sie informieren sich vor der Reise häufiger über das Reiseziel. Mottiar und Quinn (2004: 158) sprechen in diesem Zusammenhang von der „gatekeeper“-Rolle der Frauen. In der Mehrheit der Fälle entscheiden aber beide Partner gemeinsam, wobei die Frau dann häufiger allein entscheidet, wenn Kinder vorhanden sind. Begründet wird dies damit, dass die Kinderbetreuung Aufgabe der Frau ist (vgl. Fodness 1992: 12).

1 Für einen Überblick zu gemeinsamen Freizeitaktivitäten von verheirateten und unverheirateten Paaren vgl. z.B. Kalmijn und Bernasco (2000: 3). 
Das Ziel dieses Artikels ist es nicht nur zu analysieren, welcher der beiden Partner in der Beziehung eher die Entscheidungsmacht über größere Anschaffungen bzw. über die Freizeitgestaltung hat, sondern auch inwiefern diese Macht bei Partnerschaften in prekären Lebenslagen anders verteilt ist als bei besser situierten Partnerschaften.

Hierzu ziehen wir die Daten der Panelstudie „Arbeitsmarkt- und Soziale Sicherung“ (PASS) des Instituts für Arbeitsmarkt- und Berufsforschung (IAB) heran. Dieser Datensatz verfügt neben den zentral zu untersuchenden Merkmalen über einen hohen Anteil an Befragten im Arbeitslosengeld-II-Bezug und erlaubt somit belastbare statistische Analysen in diesem Bereich.

Im Rahmen dieser Untersuchung wollen wir folgende zentrale Forschungsfragen analysieren: Welcher Partner entscheidet in der Beziehung über größere Anschaffungen? Wer entscheidet über die Verwendung der gemeinsamen, freien Zeit? Und welche Faktoren sind dabei ausschlaggebend? Wie unterscheiden sich Paare in prekären Lebenslagen von relativ besser gestellten Paaren bezüglich der Verteilung der Entscheidungsmacht zwischen den beiden Partnern? ${ }^{2}$

\section{Theorie}

Entscheidungsmacht ${ }^{3}$ innerhalb von Partnerschaften lässt sich in Anlehnung an Weber (2005: 28) als Chance verstehen, innerhalb einer Partnerschaft den eigenen Willen in Bezug auf Entscheidungssituationen auch gegen Widerstreben des anderen Partners durchzusetzen. Die beiden spezifischen Formen von Entscheidungsmacht, die hier untersucht werden, klassifizieren wir nach Safilios-Rothschild (1976: 359) wie folgt (vgl. hierzu auch Lott 2009: 329): Entscheidungen über größere Anschaffungen fallen in die Kategorie der dirigierenden Macht, da es sich hierbei eher um unregelmäßig getroffene, wichtige Entscheidungen handelt. Bei Entscheidungen über die Freizeitgestaltung kann man davon ausgehen, dass hier neben der dirigierenden, auch Aspekte ausführender Macht eine Rolle spielen. Denn unter Freizeitgestaltung können sowohl regelmäßig wiederkehrende, alltägliche (z.B. die Wahl des Fernsehprogramms) wie auch unregelmäßig auftretende Freizeitaktivitäten (z.B. Urlaub oder Wochenendausflüge) verstanden werden. Demnach könnte man die Macht über die Freizeitgestaltung als die schwächere der beiden Formen verstehen, da sie einen relevanten Teil ausführender Macht beinhaltet. Für die Analysen in diesem Beitrag interessiert uns allerdings weniger die hierarchische, als die inhaltliche Unterscheidung zwischen den Machtstrukturen der beiden Entscheidungssituationen.

Als einen Ansatz zur Erklärung der Machtverhältnisse bei Entscheidungen in Partnerschaften verwenden wir den klassischen, ressourcentheoretischen Ansatz von Blood und

2 Da bisher keine Arbeiten zum Entscheidungsverhalten von Paaren im ALG-II-Bezug vorliegen, wollen wir im Folgenden eher breiter angelegt die vielfältigen Zusammenhänge zwischen Entscheidungsverhalten und verschiedenen Prädiktoren für ALG-II-Bezieher und Nicht-Bezieher im Vergleich beleuchten. Wir fokussieren unsere Analysen daher weniger auf einzelne, spezifische Hypothesen, sondern formulieren aus der Reflexion des bisherigen Forschungsstands theoretische Annahmen, die als Hintergrund dienen, vor dem die empirischen Befunde interpretiert werden.

3 Für eine ausführliche Auseinandersetzung mit Definitionen vgl. z.B. Lenz (2009) und SafiliosRothschild (1970). 
Wolfe (1978 [1960]). Diese untersuchten in ihrer Studie von 1960 anhand von acht Entscheidungen, ${ }^{4}$ wie die Macht zwischen Ehepartnern verteilt ist. Für die Struktur der Machtverhältnisse in Partnerschaften sehen sie das Verhältnis der Ressourcenausstattung zwischen den beiden Partnern als verantwortlich an. Je höher die Ressourcen des einen Partners relativ gesehen zum anderen Partner sind, umso mehr Entscheidungsmacht besitzt dieser in der Beziehung (vgl. auch Heer 1963: 137).

Während Blood und Wolfe (1978 [1960]) in ihrer Studie nur die Ehefrauen befragt haben, untersuchten Centers, Raven und Rodrigues (1971) einige Jahre später beide Ehepartner und erweiterten ihre Studie um einige Kriterien. Sie fanden heraus, dass unter anderem eine hohe Bildung und ein hoher beruflicher Status von Männern mit mehr Macht für diese in der Beziehung einhergehen.

Pahl (1983: 238) nimmt an, dass in Gesellschaften, in denen Geld gleichzeitig Macht bedeutet, die relativen ökonomischen Positionen der beiden Partner auch Einfluss auf deren Beziehung haben müssten. Je nachdem, wie die Kontrolle über die ökonomischen Ressourcen zwischen den Partnern verteilt ist, zeigt sich dies anhand des (Un-)Gleichgewichts an Macht zwischen den Partnern. Besitzt also ein Partner mehr ökonomische Ressourcen als der andere Partner, dann hat dieser Partner auch mehr Macht in der Beziehung.

Neben Bildung, Status und Einkommen verstehen wir im Rahmen dieser Studie unter

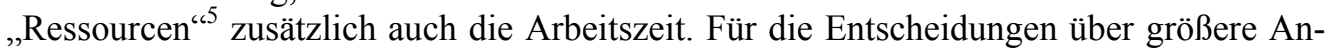
schaffungen gehen wir davon aus, dass ein Partner, der im Vergleich zum anderen Partner mehr Zeit für die Erwerbsarbeit aufbringt, eher seine Vorstellungen durchsetzen kann, da das Kapital, das für die Anschaffungen aufgewandt werden muss, vor allem auf Grund seines höheren Arbeitseinsatzes zur Verfügung steht. Für die Entscheidung über die Freizeitgestaltung vermuten wir dagegen, dass der Partner mit dem geringeren Umfang an Erwerbsarbeit eher entscheidet, da dieser über mehr Zeit für die Recherche verfügt und dem anderen gegenüber dadurch häufig einen Informationsvorsprung hat (vgl. Fodness 1992). Alternativ wäre aber auch in diesem Bereich denkbar, dass das Argument des höheren Arbeitseinsatzes im Entscheidungsprozess dominiert.

Aus dem klassischen Ressourcenansatz lässt sich folgern, dass in den meisten Fällen der Partner die höhere Entscheidungsmacht hat, der über die größere relative Ressourcenausstattung verfügt. Allerdings ist der Ressourcenansatz nicht unumstritten. Andere Studien belegen, dass die Ressourcenausstattung alleine als Erklärung für Ungleichheiten zwischen den Partnern hinsichtlich der Entscheidungsmacht nicht ausreicht. Vielmehr sollten soziale Normen und Werte ebenfalls berücksichtigt werden (vgl. Lott 2009: 351). Bereits Blumstein und Schwartz (1983) zeigen anhand der Untersuchung von Macht bei lesbischen Paaren, dass unter Berücksichtigung von Geschlechterrollen die Ressourcenverteilung zwischen den Partnern allein nicht ausschlaggebend ist.

4 (1) Welchen Job der Ehemann annehmen sollte, (2) welches Auto gekauft wird, (3) ob eine Lebensversicherung abgeschlossen wird, (4) wohin es in den Urlaub geht, (5) welches Haus oder welche Wohnung genommen wird, (6) ob die Frau arbeiten gehen oder den Job aufgeben sollte, (7) welcher Arzt zu Rate gezogen wird, wenn jemand krank wird, (8) wie viel Geld die Familie für Essen in der Woche aufwendet (vgl. Blood/Wolfe 1978 [1960]: 19).

5 Für einen Überblick familialer Ressourcen vgl. z.B. Hillmert (2002: 50f.) sowie für einen Überblick über verschiedene Ressourcen, die zwischen Partnern ausgetauscht werden können, vgl. z.B. Safilios-Rothschild (1976: 356) und Kollock/Blumstein/Schwartz (1994: 342f.). 
Nach Kirchler (1988: 328) haben Geschlechterrollen einen entscheidenden Einfluss auf das Entscheidungsverhalten in Haushalten. Er betont, dass unter anderem gesellschaftliche Normen dafür verantwortlich sind, dass es immer noch geschlechtsspezifische Unterschiede gibt. Während in der Vergangenheit der Mann die Legitimierung besaß, Entscheidungen über außerfamiliale Angelegenheiten und das Arbeitsleben zu treffen, durfte die Frau über häusliche Aktivitäten und die Kindererziehung entscheiden. Es finde, so Kirchler, ein Wandel gesellschaftlicher Normen hin zu einem egalitäreren Rollenverständnis statt. Dennoch herrschten in modernisierten westlichen Ländern trotz des Wandels immer noch gesellschaftliche Normen, die eine geschlechtsspezifische Rollenaufteilung befürworten. Männer haben dabei eine höhere Entscheidungsmacht bezüglich technischer Güter, des Autos und finanzieller Angelegenheiten inne, wohingegen Frauen vor allem bei Küchenutensilien, Kleidung, Essen und Möbeln das Sagen haben (vgl. Kirchler 1988; Pahl 1990).

Auch Nave-Herz (2004: 160) weist auf geschlechtsspezifische Unterschiede in Deutschland bezüglich der Entscheidungen in verschiedenen Bereichen hin. So entschieden Männer vor allem über größere Anschaffungen, wofür sie als Beispiele das Auto und den Rasenmäher nennt. Auch Entscheidungen, die den weiteren Bekanntenkreis und finanzielle Kapitalanlagen beträfen, lägen in der Entscheidungsmacht des Mannes. Frauen würden diesbezüglich nur beratend tätig sein. Diese seien hingegen für Entscheidungen über alltägliche Ausgaben und für Entscheidungen bezüglich der Kindererziehung verantwortlich.

Hinsichtlich einer rollentheoretischen Perspektive ist auch festzustellen, dass in der Regel den Frauen in der Familie die Rolle des „kinkeeper“ zuteilwird. Deren Aufgabe ist es, die einzelnen Familienmitglieder zusammenzuführen, so dass diese miteinander in Kontakt bleiben (vgl. Kranichfeld 1987; Rosenthal 1985). Diese Rolle könnte womöglich auch auf Paarbeziehungen im Allgemeinen übertragen werden. Gerade was die Gestaltung der gemeinsamen Freizeit betrifft, so kann vermutet werden, dass Frauen dadurch den Zusammenhalt und die Stabilität der Beziehung stärken wollen.

$\mathrm{Zu}$ den Auswirkungen des Arbeitslosengeld-II-Bezugs als prekäre Lage von Partnerschaften gibt es derzeit weder empirische Befunde, noch theoretische Überlegungen. In Anknüpfung an die Rollentheorie (vgl. Goffman 2001) könnte man vermuten, dass in diesen Fällen die gelebten Rollenarrangements nicht mehr in gleicher Weise fortgesetzt werden können wie sie in nicht bedürftigen Haushalten noch immer häufig vorzufinden sind. Das gilt insbesondere für das klassische Male-Breadwinner-Modell. Wie Brehmer, Klenner und Klammer (2010) für Deutschland zeigen, sind Familienernährerinnen häufig bei Paaren in Niedrigeinkommenshaushalten zu finden. Schon Stamp (1985) wie auch Tichenor (1999) stellen für Familienernährerinnen bei sozio-ökonomisch besser gestellten Paaren fest, dass die Frau bei einem großen Teil der befragten Paare alleine das Geld verwaltet.

In Anknüpfung an neuere Gendertheorien (vgl. Lorber 1999; Lorber/Farrell 1991; West/Fenstermaker 1995; West/Zimmerman 1987, 2002) kann man das Geschlechterverhältnis unabhängig vom biologischen Geschlecht vielmehr als alltägliche Herstellungsleistung in der Interaktion der beiden Partner verstehen. Geht man davon aus, dass in der Gruppe der ALG-II-Leistungsbezieher traditionelle Rollenmuster in der Partnerschaft häufiger nicht mehr reproduziert werden können als unter den Nicht-Beziehern, etwa weil der Mann seiner Ernährerrolle nicht mehr nachkommen kann, könnte dies auch eine Verschie- 
bung der Machtverhältnisse nach sich ziehen. Der Mann würde in diesem Fall an Macht verlieren, da er durch sein Verhalten traditionelle Aspekte von „Männlichkeit“ nicht mehr reproduzieren kann durch die ihm eine höhere Entscheidungsmacht zukam. Im Gegenteil kann es bei Partnerschaften im Leistungsbezug - wie erwähnt - häufiger dazu kommen, dass die Frau sich „männlich“ verhält, indem sie als Einzige durch eine Erwerbstätigkeit zum Haushaltseinkommen beiträgt. Daraus lässt sich im Umkehrschluss ableiten, dass in diesen Fällen Frauen über größere Entscheidungsmacht verfügen müssten. Nach West und Zimmerman (2002: 22) könnte die Frau allerdings ein Interesse daran haben dem Mann weiterhin eine rollenkonforme Geschlechtsdarstellung zu ermöglichen, da sie dadurch selbst in die Lage versetzt wird sich konform zu verhalten und ihre Geschlechtsidentität zu bestätigen. Auf Grund der besonders prekären sozio-ökonomischen Lage im ALG-IIBezug vermuten wir jedoch eher, dass es zumindest zu einer teilweisen Aufweichung von traditionellen Geschlechterrollenarrangements kommt.

Diese Vermutung verstärkt sich, wenn man den institutionellen Druck im ALG-II-Kontext berücksichtigt. So soll etwa bei der sanktionsbewährten Verpflichtung zur Aufnahme einer zumutbaren Erwerbsarbeit keine explizite Geschlechterdifferenzierung vorgenommen werden. Es kann angenommen werden, dass dies in der Tendenz zu einer Egalisierung der Geschlechterverhältnisse führt. Auch wenn einzelne, jüngere Befunde nicht darauf hindeuten, dass es im Rahmen der Umsetzung des SGB II bisher tatsächlich zu einer substanziellen Nivellierung von Geschlechterungleichheiten gekommen ist (Jaehrling 2010; Rudolph 2010), wollen wir dies mit den vorliegenden Daten noch eingehender prüfen.

\section{Daten und Methode}

Grundlage der quantitativen Analysen ist das Panel „Arbeitsmarkt und soziale Sicherung“ (PASS) des Instituts für Arbeitsmarkt- und Berufsforschung (IAB). Die Befragung wird seit Ende 2006 jährlich durchgeführt und beinhaltet Angaben von 8.000 bis 12.000 Haushalten in Deutschland (Berg et al. 2011).

Das Stichprobendesign erlaubt neben einer Hochrechnung auf die Wohnbevölkerung in Deutschland detaillierte Analysen von finanziell bedürftigen Haushalten, da die Hälfte der Stichprobe aus dem Bestand der Bezieher von Arbeitslosengeld II gezogen wurde. Dies ist im Kontext der vorliegenden Fragestellung wichtig, um auf eine ausreichende Fallzahl von Paaren im Niedrigeinkommensbereich zurückgreifen und somit valide statistische Aussagen treffen zu können.

Ein weiterer zentraler Vorteil des PASS für unsere Fragestellung gegenüber anderen Studien ist das Prinzip, den kompletten Haushalt, d.h. alle Mitglieder über 15 Jahren, persönlich zu befragen. Dies eröffnet die Möglichkeit, die Antworten beider Partner getrennt zu analysieren und Unterschiede im Antwortverhalten berücksichtigen zu können.

Für die vorliegenden Auswertungen greifen wir ausschließlich auf die zweite Erhebungswelle der Befragung zurück, da bisher nur dort die Fragen nach der Verteilung der Entscheidungsmacht zwischen den Partnern gestellt wurden. Unsere Analysen können somit nur im Querschnitt durchgeführt werden. Auch wenn so keine Aussagen über kausale Effekte gemacht werden können, ergeben sich aufschlussreiche Eindrücke über die Zusammenhänge der Machtallokation in Partnerschaften. 
Neben der deskriptiven Auswertung verwenden wir multivariate Regressionsmodelle, um zu prüfen, inwiefern sich Zusammenhänge zwischen den Antworten zur Machtverteilung und zentralen unabhängigen Variablen auch noch unter Kontrolle weiterer relevanter Merkmale zeigen oder ob sie auf Grund von Kompositionseffekten des Analysesamples verschwinden. Die Analyseeinheit ist das Individuum, so dass immer beide Partner eines Paares als einzelne Fälle in die Berechnungen eingehen.

Das Analysesample umfasst 2.532 heterosexuelle, in einem Haushalt zusammenlebende Paare und somit 5.064 Einzelpersonen. Davon beziehen 579 Paare zum Befragungszeitpunkt ALG II. ${ }^{6}$ Die Aufteilung der Entscheidungsmacht hinsichtlich größerer Anschaffungen sowie der Freizeitgestaltung wurde in der Befragung mit zwei Fragen operationalisiert. Auf die Fragen

„Wer hat in Ihrer Partnerschaft oder in Ihrer Ehe das letzte Wort, wenn Entscheidungen über größere Anschaffungen getroffen werden? “

und

„Wer entscheidet in Ihrer Partnerschaft oder in Ihrer Ehe im Allgemeinen darüber, wie Sie Ihre gemeinsame Freizeit verbringen?"

konnten die Befragten mit den Kategorien „Immer ich“, „Eher ich“, „Eher mein Partner/ meine Partnerin“ und „Immer mein Partner/meine Partnerin“ antworten. Wenn die Befragten von sich aus „Beide in gleichem Maße/abwechselnd“ genannt haben, wurde diese Antwort entsprechend erfasst, obwohl die Antwortkategorie nicht als Option vorgelesen wurde. Dadurch wurden die Befragten eher angehalten, eine Richtung anzugeben. Damit soll das sozial erwünschte Antwortverhalten reduziert werden. Wir sehen hier keinen Anhaltspunkt, starke systematische Verzerrungen in die eine oder andere Richtung zu vermuten. Zumindest für eine höhere Anzahl an Antwortverweigerungen finden wir keine Belege, da der Anteil der Antwortverweigerungen bei der Frage zu den größeren Anschaffungen bei lediglich $0,17 \%$ und bei der Frage zu der Freizeitgestaltung bei 0,31\% liegt. Letztlich lässt sich mit den vorliegenden Daten jedoch nicht vollständig klären, ob es Verzerrungen im Antwortverhalten durch die Art der Fragestellung gibt.

Welche größeren Anschaffungen und welche Freizeitaktivitäten dabei explizit gemeint sind, wurde im Fragebogen nicht näher erklärt. Es wurde damit der individuellen Einschätzung der Befragten überlassen, was sie darunter verstehen. Man kann dennoch vermuten, dass unter der Formulierung ,größere Anschaffungen“ keine Güter des alltäglichen Bedarfs wie beispielsweise Essen verstanden werden. Allerdings ist auch zu berücksichtigen, dass bei ALG-II-Empfängern „größere Anschaffungen“ vermutlich andere Güter umfassen als bei Nicht-Beziehern. Da wir keine genaueren Informationen zum Verständnis der Befragten haben, müssen wir vereinfachend davon ausgehen, dass sie für sich relevante Entscheidungssituationen als Referenz für ihre Antwort angenommen haben.

Für die Analyse bilden wir die Machtverteilung zwischen den Partnern mit einer dreistufigen Variable mit den Ausprägungen „Mann entscheidet eher“, „beide entscheiden

6 Für 12 Paare konnte nicht bestimmt werden, ob ein Bezug vorliegt. Sie entfallen somit aus den multivariaten Analysen. 
gemeinsam“ und „Frau entscheidet eher“ ab. Dabei handelt es sich um eine geschlechtsspezifische Rekodierung der fünfstufigen Ausgangsmerkmale. ${ }^{7}$

Zur Operationalisierung von Einflussvariablen, die sich aus den ressourcentheoretischen Überlegungen ergeben, verwenden wir das individuelle Bildungsniveau nach der dreistufigen CASMIN-Skala (vgl. Brauns/Steinmann 1999) und die Bildungsdifferenz zwischen den Partnern. ${ }^{8}$

Daneben wird der Unterschied in der finanziellen Ressourcenausstattung beider Partner über die Kategorien „Mann verfügt über höheres Nettoeinkommen“, „gleiche Nettoeinkommen“ und „Frau verfügt über höheres Nettoeinkommen“ abgebildet. Dazu vergleichen wir die individuellen Nettoeinkommen der Partner aus einer evtl. Erwerbstätigkeit, wobei wir eine Differenz von einem Euro bereits als Unterschied ansehen. Das Einkommensniveau wird gleichzeitig über die Summe beider Nettoeinkommen als metrische Variable kontrolliert.

Nach der Ressourcentheorie sollte neben dem Einkommen auch der sozio-ökonomische Status eine Rolle spielen, der sich aus der individuellen Erwerbstätigkeit ergibt. Deshalb berücksichtigen wir den Unterschied im ISEI-Wert (International Socio-Economic Index of Occupational Status) zwischen den Partnern (vgl. Ganzeboom/Treiman 2003). Dies bilden wir analog zum Einkommen über die Differenz im Status der Partner sowie über das Niveau der summierten Statusangaben ab.

In Abschnitt 2 haben wir außerdem argumentiert, dass die Ressourcen auch über die aufgewandte Erwerbsarbeitszeit abgebildet werden sollten. Dies geschieht in unseren Modellen ebenfalls über die Differenz der Arbeitszeiten beider Partner und die Summe über beide Partner. Wir greifen dabei auf die angegebene tatsächliche, wöchentliche Arbeitszeit zurück.

Im Kontext der Ressourcentheorie wird oft die Kontoführung als Indikator für Machtallokation angesehen (vgl. Kirchler 1988; Lott 2009). Wir nehmen die Führung von gemeinsamen bzw. individuellen Konten daher als Kovariate mit in unsere Analysemodelle auf.

Die rollentheoretischen Annahmen operationalisieren wir zum einen über die Abfrage der Einstellung zu den Geschlechterrollen. Dazu greifen wir auf die Zustimmungsfrage „Die Aufgabe des Ehemannes ist es, Geld zu verdienen, die der Ehefrau, sich um den Haushalt und die Familie zu kümmern“ zurück, die über eine Skala von 1 „Stimme voll und ganz zu“ bis 4 „Stimme überhaupt nicht zu“ abgefragt wird. ${ }^{9}$

Zum anderen verwenden wir als weiteres Maß für eine traditionelle Rolleneinstellung die Religiosität der befragten Personen (vgl. z.B. Kalmijn/Bernasco 2000). Hierzu haben

7 Details zur Umsetzung der Datenaufbereitung und Durchführung der Analysen können den STATA do-Files entnommen werden, die die Autoren auf Anfrage zur Verfügung stellen.

8 Deskriptive Auszählungen zu allen für die Analysen verwendeten Merkmalen lassen sich den Tabellen 4, 5, 6 und 7 im Anhang entnehmen.

9 Dieses Item ist Teil einer Batterie mit vier Fragen zu Meinungen zum Verhältnis von Familie und Beruf. Eine Indexbildung haben wir geprüft, auf Grund der Ergebnisse der Reliabilitäts- und Faktoranalysen dann aber verworfen. Das verwendete Item bildet die Modernität der Rolleneinstellung unserer Ansicht nach am besten ab. Trotz der geringen Anzahl an Ausprägungen nehmen wir die Variable als metrisch mit in unsere Modelle auf, da wir davon ausgehen, dass das zugrunde liegende Konstrukt metrisch skaliert ist und die Variable lediglich eine vergröberte Messung dieses Konstrukts darstellt. 
wir die Frage „Unabhängig davon, ob Sie einer bestimmten Religionsgemeinschaft angehören, für wie religiös halten Sie sich? Für ,Überhaupt nicht religiös', für ,Eher nicht religiös', für ,Eher religiös“ oder für ,Sehr religiös‘ “" verwendet. Wir gehen davon aus, dass ein hohes $\mathrm{Ma}$ an Religiosität unabhängig von einer bestimmten Konfessionszugehörigkeit mit einer eher traditionellen Rolleneinstellung einhergeht.

Bei der Analyse von Partnerschaften erscheint es wesentlich, die familiäre Situation zu kontrollieren (vgl. z.B. Kalmijn/Bernasco 2000; Vogler 2005). Unterschieden wird, ob es sich bei den betrachteten Paaren um Ehepaare oder nichteheliche Lebensgemeinschaften handelt. Außerdem kontrollieren wir, ob mindestens ein Kind unter 15 Jahren im Haushalt lebt. Es ist zu vermuten, dass in Haushalten mit Kindern Frauen stärker entscheiden, weil ihnen häufig noch die Aufgabe der Kinderbetreuung und damit auch die Vertretung der Interessen des Kindes im Entscheidungsprozess zukommt.

Als Proxy für das Familienklima nehmen wir die Frage „Wie häufig gibt es in Ihrem Haushalt Missverständnisse, Spannungen oder Konflikte? ,Sehr häufig‘, ,Häufig ‘, ,Manchmal', ,Selten“ oder ,Sehr selten oder nie" ?" auf. Bei diesem Merkmal kehren wir jedoch die Kodierung um, so dass sich der zugehörige Koeffizient in den Modellen intuitiver interpretieren lässt. Wir gehen tendenziell davon aus, dass ein schlechtes Familienklima eher dazu führt, dass die Partner getrennt entscheiden, da es im Konfliktfall schwieriger erscheint zu Konsensentscheidungen zu kommen.

Geschlecht und Alter sowie den Migrationshintergrund kontrollieren wir auf der Individualebene. Als eine Person mit Migrationshintergrund wird jemand definiert, der selbst oder dessen Eltern nach Deutschland zugezogen sind. Auf Ebene der Dyaden kommen die Altersdifferenz zwischen den Partnern (,Mann älter“, „,beide gleich alt" und „Frau älter") (zur Messung von kultureller Homogamie, beispielsweise bezüglich der Altersdifferenz von Paaren, vgl. z.B. Kalmijn/Bernasco 2000: 10f.) und der Wohnort in den neuen oder alten Bundesländern hinzu. Ebenso kontrollieren wir auf dieser Ebene die Migrationskonstellation, d.h. ob kein Partner, nur der Mann, nur die Frau oder beide Partner einen Migrationshintergrund haben.

Für die multivariaten Berechnungen verwenden wir auf Grund der nominalen Skalenniveaus der abhängigen Variablen multinomiale Logit-Modelle, wobei „beide entscheiden gemeinsam" die Referenzkategorie bildet. Dadurch ist es möglich, die Zusammenhänge für die Entscheidungsmacht des Mannes von denen der Frau zu trennen und dennoch ein gemeinsames Modell zu schätzen. Dabei wird das in Gleichung 1 angegebene Modell verwendet, um die Wahrscheinlichkeit für die Antwortmöglichkeiten $\operatorname{Pr}\left(f_{i j}\right)$ zu schätzen (siehe z.B. Skrondal/Rabe-Hesketh 2004: 29).

$$
\operatorname{Pr}\left(f_{i j}\right)=\exp \left(V_{i j}^{f}\right) / \sum_{\mathrm{a}=1}^{\mathrm{A}} \exp \left(V_{i j}^{a}\right)
$$

Da die beiden Partner im von uns betrachteten Kontext nicht unabhängig voneinander agieren, muss der Interdependenz in der jeweiligen Paardyade Rechnung getragen werden (vgl. Kenny/Kashy/Cook 2006). Wir berücksichtigen dies, indem wir in den linearen Prädiktor $V^{a}{ }_{i j}$ für die Antwortmöglichkeit $a$ mit $\gamma_{j 0}^{a}$ einen ,random intercept“" auf der Dyadenebene aufnehmen (vgl. Rabe-Hesketh/Skrondal 2004: 111). Mit dieser paarspezifischen Konstante kann die Interdependenz des Antwortverhaltens der Partner in der Paardyade explizit modelliert und damit kontrolliert werden. 


$$
V^{a}{ }_{i j}=\beta^{a}{ }_{0}+\boldsymbol{\beta}^{a} \mathbf{x}_{i j}+\gamma^{a}{ }_{j 0}+\boldsymbol{\gamma}^{a} \mathbf{z}_{j}
$$

Gleichung 2 stellt das Modell für den linearen Prädiktor dar. Mit $\beta^{a}{ }_{0}$ wird darin der konstante Term des Modells bezeichnet. $\mathbf{x}_{i j}$ und $\boldsymbol{\beta}^{a}$ sind Vektoren und stehen für alle auf der Ebene der individuellen Partner variierenden, unabhängigen Variablen, respektive die dazugehörigen Regressionskoeffizienten. Analog dazu stehen $\mathbf{z}_{j}$ und $\gamma^{a}$ für die Vektoren der unabhängigen Variablen, die nur auf der Dyadenebene variieren bzw. deren jeweilige Koeffizienten.

Zur Unterscheidung der Zusammenhänge zwischen der Gruppe der ArbeitslosengeldII-Bezieher und der Nicht-Bezieher führen wir für alle erklärenden Variablen einen Interaktionsterm mit der Indikatorvariable „Arbeitslosengeld-II-Bezug: ja/nein“ in die Modelle ein. Dadurch wird es möglich - ähnlich zu getrennten Regressionsmodellen - gruppenspezifische Koeffizienten für die Zusammenhänge der unabhängigen Variablen mit der Entscheidungsmacht zu schätzen. Dies ist möglich, indem man den Haupteffekt (der als Effekt in der Referenzgruppe interpretiert werden kann) und den Interaktionskoeffizienten addiert. Daraus ergibt sich der Effekt in der Vergleichsgruppe. Wir geben die Schätzungen für beide Gruppen als eigene Spalten in den Modelltabellen aus. Zur korrekten Spezifikation des Modells haben wir neben den Interaktionstermen auch den Haupteffekt für den ALG-II-Bezug aufgenommen. Um die Zusammenhangsstrukturen in den beiden Gruppen mit und ohne ALG-II-Bezug jeweils vollständig darstellen zu können, haben wir die Aufnahme von Interaktionstermen nicht von einem statistisch signifikanten $p$-Wert abhängig gemacht. Unsere Interpretation orientiert sich daran, ob die Effekte innerhalb der jeweiligen Gruppen statistisch signifikant sind oder nicht.

\section{Ergebnisse}

Generell zeigt sich in der deskriptiven Betrachtung, dass das egalitäre Entscheidungsmodell mit Abstand am häufigsten vorkommt. Dies gilt sowohl für die Entscheidungen über größere Anschaffungen $(79,25 \%$, vgl. Tabelle 1) als auch für die Freizeitgestaltung $(81,75 \%)$. Kleinere Unterschiede sind erkennbar, wenn man in der jeweiligen Entscheidungssituation Paare mit und ohne ALG-II-Bezug vergleicht. Bezüglich größerer Anschaffungen entscheiden Paare mit ALG-II-Bezug etwas seltener gemeinsam. Dabei ist auffällig, dass die Frauen in diesen Partnerschaften mehr Entscheidungsmacht haben. Dies geht vor allem auf Kosten der gemeinsamen Entscheidungen. Bei Männern lassen sich hingegen nur geringfügige Unterschiede feststellen.

Bei den Entscheidungen über die Freizeitgestaltung ergibt sich im Vergleich der Gruppen mit und ohne ALG II das gegenteilige Bild: Bedürftige Paare mit Leistungsbezug entscheiden in diesem Fall häufiger gemeinsam als Paare in gesicherteren finanziellen Verhältnissen. Während sich Männer in Partnerschaften mit ALG-II-Bezug nicht von Männern ohne Bezug unterscheiden, verfügen die Frauen über weniger Entscheidungsmacht, wenn sie Leistungen beziehen. 
Tabelle 1: Entscheidungsmacht bei Paaren mit und ohne Arbeitslosengeld-II-Bezug (individuelle Antworten der Zielpersonen)

\begin{tabular}{|c|c|c|c|c|c|c|}
\hline & \multicolumn{4}{|c|}{ Arbeitslosengeld-II-Bezug } & & \\
\hline & \multicolumn{2}{|c|}{ ohne } & \multicolumn{2}{|c|}{ mit } & \multicolumn{2}{|c|}{ Gesamt } \\
\hline & $\%$ & $n$ & $\%$ & $n$ & $\%$ & $n$ \\
\hline \multicolumn{7}{|c|}{ Entscheidungen über größere Anschaffungen } \\
\hline Mann entscheidet eher & 12,61 & 442 & 11,53 & 132 & 12,57 & 132 \\
\hline beide entscheiden gemeinsam & 79,40 & 3.024 & 75,85 & 831 & 79,25 & 3.855 \\
\hline Frau entscheidet eher & 7,99 & 335 & 12,62 & 164 & 8,18 & 164 \\
\hline Gesamt & 100,00 & 3.801 & 100,00 & 1.127 & 100,00 & 4.928 \\
\hline \multicolumn{7}{|c|}{$\chi^{2}($ gewichtet $)=14,3 ; P($ gewichtet $)=0,105 ; \chi^{2}$ (ungewichtet $)=32,0 ; P($ ungewichtet $)=0,000$} \\
\hline \multicolumn{7}{|c|}{ Entscheidungen über die Freizeitgestaltung } \\
\hline Mann entscheidet eher & 5,78 & 195 & 5,79 & 68 & 5,78 & 263 \\
\hline beide entscheiden gemeinsam & 81,63 & 3.095 & 84,53 & 946 & 81,75 & 4.041 \\
\hline Frau entscheidet eher & 12,59 & 512 & 9,68 & 110 & 12,47 & 622 \\
\hline Gesamt & 100,00 & 3.802 & 100,00 & 1.124 & 100,00 & 4.926 \\
\hline
\end{tabular}

$\chi^{2}($ gewichtet $)=3,9 ; P($ gewichtet $)=0,559 ; \chi^{2}($ ungewichtet $)=11,5 ; P($ ungewichtet $)=0,003$

$\%=$ Anteilswert in Prozent (gewichtet); $n=$ Absolutwert (ungewichtet)

Quelle: Eigene Berechnungen (PASS SUF 2006-2010 V2, Welle 2)

\subsection{Multivariate Modellierung der Machtverteilung bei größeren Anschaffungen}

Der zentrale Untersuchungsgegenstand dieses Beitrags ist der Vergleich von Paaren im ALG-II-Bezug mit solchen ohne Bezug hinsichtlich der Verteilung der Entscheidungsmacht zwischen den Partnern. Dieser wird insbesondere unter Kontrolle der Ressourcenverteilung und Rolleneinstellungen analysiert, da sich in vorangegangenen Studien gezeigt hat, dass diese Aspekte wesentlichen Einfluss auf die Machtverteilung nehmen (vgl. Lott 2009; Ludwig-Mayerhofer/Gartner/Allmendinger 2006; Vogler 1998). Wir betrachten zuerst die Entscheidungsmacht hinsichtlich größerer Anschaffungen (vgl. Tabelle 2).

Die Einstellungen der befragten Personen zu den Geschlechterrollen zeigen, dass sowohl für Männer als auch für Frauen die individuelle Entscheidungsmacht mit steigender Modernität der Rollenbilder zugunsten einer egalitären Verteilung abnimmt. Dies gilt sowohl unter Arbeitslosengeld-II-Beziehern als auch unter den Nicht-Beziehern. Für die Religiosität, die wir ebenfalls als Proxy für eine traditionelle Einstellung verwenden, ergeben sich dagegen keine statistisch signifikanten Effekte. 
Tabelle 2: Regressionsmodell - Entscheidung über größere Anschaffungen

\begin{tabular}{|c|c|c|c|c|}
\hline & \multicolumn{2}{|c|}{ kein ALG-II-Bezug } & \multicolumn{2}{|c|}{ ALG-II-Bezug } \\
\hline & $\begin{array}{l}\text { Mann } \\
\text { entscheidet } \\
\text { eher }\end{array}$ & $\begin{array}{l}\text { Frau } \\
\text { entscheidet } \\
\text { eher }\end{array}$ & $\begin{array}{l}\text { Mann } \\
\text { entscheidet } \\
\text { eher }\end{array}$ & $\begin{array}{l}\text { Frau } \\
\text { entscheidet } \\
\text { eher }\end{array}$ \\
\hline \multicolumn{5}{|l|}{ individuelle Merkmale } \\
\hline weiblich & $-0,693^{* * *}$ & 0,049 & $-0,235$ & $0,488^{*}$ \\
\hline Alter & $-0,031^{\star \star *}$ & $-0,020^{*}$ & $-0,021$ & $-0,016$ \\
\hline niedrige Bildung & 0,079 & $-0,235$ & $-0,866^{* *}$ & $-0,712^{*}$ \\
\hline mittlere Bildung & \multicolumn{2}{|l|}{ Referenzkategorie } & \multicolumn{2}{|l|}{ Referenzkategorie } \\
\hline hohe Bildung & $0,385+$ & 0,056 & 0,047 & 0,268 \\
\hline $\begin{array}{l}\text { Migrationshintergrund } \\
\text { Missverständnisse, Spannungen oder Konflikte im } \\
\text { Haushalt }\end{array}$ & $\begin{array}{l}-0,517 \\
0,363^{* * *}\end{array}$ & $\begin{array}{l}0,226 \\
0,410^{\star * *}\end{array}$ & $\begin{array}{l}0,520 \\
0,223\end{array}$ & $\begin{array}{r}-0,179 \\
0,103\end{array}$ \\
\hline gemeinsames Konto & \multicolumn{2}{|l|}{ Referenzkategorie } & \multicolumn{2}{|l|}{ Referenzkategorie } \\
\hline eigenes Konto & $0,380^{*}$ & $0,347+$ & $0,794^{*}$ & $0,741^{*}$ \\
\hline weder noch & $-0,629$ & 0,584 & 0,256 & 0,486 \\
\hline $\begin{array}{l}\text { Einstellung der Zielperson zu Geschlechterrollen } \\
\text { Religiosität }\end{array}$ & $\begin{array}{l}-0,499^{* * *} \\
0,062\end{array}$ & $\begin{array}{l}-0,287^{* *} \\
-0,108\end{array}$ & $\begin{array}{l}-0,379^{* *} \\
0,237\end{array}$ & $\begin{array}{l}-0,368^{* *} \\
-0,159\end{array}$ \\
\hline \multicolumn{5}{|l|}{ dyadische Merkmale } \\
\hline Mann 0 bis 5 Jahre älter & \multicolumn{2}{|l|}{ Referenzkategorie } & \multicolumn{2}{|l|}{ Referenzkategorie } \\
\hline Mann mehr als 5 Jahre älter & 0,236 & 0,092 & $-0,272$ & $-0,158$ \\
\hline Mann jünger & $-0,140$ & $-0,289$ & $-0,574$ & 0,258 \\
\hline $\begin{array}{l}\text { Mann höher gebildet } \\
\text { gleiches Bildungsniveau }\end{array}$ & $\begin{array}{l}0,182 \\
\text { Referenzkategorie }\end{array}$ & $-0,308$ & $\begin{array}{l}0,161 \\
\text { Referenzkategorie }\end{array}$ & 0,042 \\
\hline Frau höher gebildet & $-0,271$ & 0,234 & $-0,132$ & 0,275 \\
\hline Wohnort in den neuen Bundesländern & 0,134 & 0,040 & $-0,158$ & $-0,329$ \\
\hline kein Migrationshintergrund & \multicolumn{2}{|l|}{ Referenzkategorie } & \multicolumn{2}{|l|}{ Referenzkategorie } \\
\hline nur Mann Migrationshintergrund & 0,465 & 0,036 & $-0,435$ & 0,678 \\
\hline nur Frau Migrationshintergrund & $0,800^{*}$ & 0,114 & 0,390 & 0,527 \\
\hline beide Migrationshintergrund & 0,214 & $-0,247$ & $-0,264$ & 0,012 \\
\hline $\begin{array}{l}\text { Ehepaar } \\
\text { mindestens } 1 \text { Kind unter } 15 \text { Jahren im Haushalt }\end{array}$ & $\begin{array}{l}-0,010 \\
-0,402^{*}\end{array}$ & $\begin{array}{l}-0,099 \\
-0,082\end{array}$ & $\begin{array}{l}-0,304 \\
-0,620+\end{array}$ & $\begin{array}{l}-0,173 \\
-0,422\end{array}$ \\
\hline $\begin{array}{l}\text { Mann hat höheres Nettoeinkommen } \\
\text { gleiche Nettoeinkommen }\end{array}$ & $\begin{array}{l}-0,289 \\
\text { Referenzkategorie }\end{array}$ & $-0,629$ & Referenzkategorie & 2,164 \\
\hline Frau hat höheres Nettoeinkommen & $-0,560$ & $-0,555$ & 0,163 & $-1,695$ \\
\hline Summe Nettoeinkommen beider Partner & 0,000 & 0,000 & $-0,001$ & 0,000 \\
\hline $\begin{array}{l}\text { Mann hat höheren sozio-ökonomischen Status } \\
\text { gleicher Status }\end{array}$ & \multicolumn{2}{|l|}{ Referenzkategorie } & \multicolumn{2}{|l|}{ Referenzkategorie } \\
\hline Frau hat höheren sozio-ökonomischen Status & 0,098 & $0,744+$ & $-0,084$ & 3,285 \\
\hline Summe sozio-ökonomischer Status beider Partner & $-0,013^{\star *}$ & $-0,015^{\star *}$ & $-0,013$ & $-0,039^{*}$ \\
\hline $\begin{array}{l}\text { Mann hat längere Arbeitszeit } \\
\text { gleiche Arbeitszeit }\end{array}$ & Referenzkategorie & 0,122 & \multicolumn{2}{|c|}{ Referenzkategorie } \\
\hline Frau hat längere Arbeitszeit & 0,061 & $-0,242$ & $-0,947$ & $-0,130$ \\
\hline Summe Arbeitszeit beider Partner & 0,007 & $0,011^{*}$ & 0,026 & $-0,009$ \\
\hline Arbeitslosengeld-II-Bezug & $-0,661$ & $-0,882$ & & \\
\hline Konstante & $-0,626$ & $-1,765^{*}$ & $-1,287$ & $-2,647$ \\
\hline
\end{tabular}

$\mathrm{N}=4.506 ; * * *: \mathrm{p}<0,001 ; * *: \mathrm{p}<0,01 ; *: \mathrm{p}<0,05 ;+\mathrm{p}<0,1$

Regressionskoeffizienten eines multinomialen logit-Modells mit random intercept auf der Paarebene; Basiskategorie: „beide in gleichem Maße/abwechselnd“

Quelle: Eigene Berechnungen (PASS SUF 2006-2010 V2, Welle 2) 
Lebt in einem Haushalt ohne ALG-II-Bezug ein Kind unter 15 Jahren, entscheidet seltener der Mann alleine über größere Anschaffungen. Dies deutet darauf hin, dass eine klassische Rollenaufteilung bzgl. der Kinderbetreuung Einfluss auf die Entscheidungsmacht zwischen den Partnern nimmt, da die Frau, die häufiger mit der Kinderbetreuung betraut ist, relativ zum Mann stärker mitentscheidet. Für Haushalte mit Kindern im ALG-IIBezug lässt sich dieser Befund allerdings nicht bestätigen. Für die Institutionalisierung der Partnerschaft durch eine Ehe findet man keinen Zusammenhang mit der Verteilung der Entscheidungsmacht. In Haushalten ohne ALG-II-Bezug korreliert das Familienklima stark mit dem Entscheidungsverhalten. Je häufiger Missverständnisse, Spannungen oder Konflikte im Haushalt auftreten, umso häufiger wird getrennt entschieden. Dies lässt sich für Haushalte mit ALG-II-Bezug nicht bestätigen. Unter Umständen sind in diesen Partnerschaften Konflikte nicht so einflussreich, da die Partner auf Grund äußerer Umstände wie der prekären Lage und dem institutionellen Druck stärker gezwungen sind, gemeinschaftlich Lösungen zu finden.

Der Einfluss von Ressourcen, die mit der Erwerbstätigkeit zusammenhängen, ist in unseren Modellen oft nur schwach ausgeprägt. Ein hoher gemeinsamer sozio-ökonomischer Status als Summe aus beiden Erwerbstätigkeiten ist häufiger mit gemeinsamen Entscheidungsprozessen verknüpft. Bei den Partnerschaften ohne ALG II zeigt sich dies sowohl durch die niedrigere alleinige Entscheidungsmacht des Mannes als auch der Frau. Unter den ALG II beziehenden Paaren weist zumindest die geringere Entscheidungsmacht der Frau in diese Richtung. Daneben hat lediglich noch das Niveau der insgesamt aufgewandten Erwerbsarbeitszeit einen statistisch signifikanten Zusammenhang. Die Frau entscheidet in Nicht-ALG-II-Partnerschaften häufiger alleine über größere Anschaffungen, wenn das Niveau der insgesamt erbrachten Arbeitszeit hoch ist. Einen deutlichen Einfluss hat die getrennte Kontoführung innerhalb der Partnerschaft. Führt bei den Nicht-ALG-IIPaaren ein eigenes Konto zumindest für den Mann häufiger zu einer alleinigen Entscheidungsmacht, zeigt sich dieser Zusammenhang im ALG-II-Bezug für beide Partner.

Ein niedriges Bildungsniveau der befragten Person führt in ALG-II-Partnerschaften häufig dazu, dass eher ein egalitäres Entscheidungsmodell berichtet wird. Dies ist bei Paaren ohne Leistungsbezug nicht der Fall. Man kann vermuten, dass Personen mit einem höheren Bildungsniveau auf Grund des eigentlich geringeren Risikos den Eintritt in den ALG-II-Bezug als noch belastender erleben, was sich auch negativ auf die Fähigkeit zur gemeinschaftlichen Entscheidungsfindung auswirkt. Außerdem kann man davon ausgehen, dass diese oft erst seit kurzer Zeit im Bezug sind und daher noch kaum Möglichkeiten hatten, Lösungsstrategien zu entwickeln. Differenzen im Bildungsniveau der beiden Partner haben dagegen in keiner der beiden Gruppen einen Einfluss.

Grundsätzlich neigen die Befragten dazu, dem eigenen Geschlecht eine höhere Entscheidungsmacht zuzuschreiben. Dies deutet darauf hin, dass ein Unterschied in der Einschätzung der Machtverhältnisse zwischen den Partnern besteht. Das Alter der Befragten hat unter den Nicht-Beziehern einen egalisierenden Effekt. Ältere Paare entscheiden häufiger gemeinsam als jüngere. Dies lässt nicht zwangsläufig auf einen echten Alterseffekt schließen, da ältere Personen in der Tendenz auch in länger andauernden Partnerschaften leben. Für die Dauer der Partnerschaft können wir mit den vorliegenden Daten aber leider nicht kontrollieren. Die Lage des Wohnortes in den neuen oder alten Bundesländern wirkt sich nicht auf die Verteilung der Entscheidungsmacht aus. Der individuelle 
Migrationshintergrund wirkt sich nicht statistisch signifikant auf das Entscheidungsverhalten aus. Allerdings hat die Migrationskonstellation innerhalb von Nicht-ALG-IIPaaren dann einen Einfluss, wenn nur die Frau, nicht jedoch der Mann einen Migrationshintergrund hat. In diesen Fällen entscheidet der Mann häufiger alleine über größere Anschaffungen.

\subsection{Multivariate Modellierung der Machtverteilung bei der Freizeitgestaltung}

Neben der Verteilung der Entscheidungsmacht bezüglich größerer Anschaffungen betrachten wir als weiteren Aspekt der Machtverteilung innerhalb von Partnerschaften die Entscheidung über die Gestaltung der Freizeit. Dafür haben wir die gleichen statistischen Modelle gerechnet und lediglich die abhängige Variable ersetzt (vgl. Tabelle 3).

Modernere Geschlechterrolleneinstellungen gehen bei Paaren ohne ALG-II-Bezug häufiger mit einem Machtverlust des Mannes einher. In der Gruppe der ALG-II-Bezieher lässt sich hier kein Zusammenhang nachweisen. In eine ähnliche Richtung deutet der Einfluss der Religiosität der befragten Person, wenn man diese Information ebenfalls als einen Hinweis auf ein traditionelles Rollenverständnis interpretiert. Je religiöser eine befragte Person ist, umso häufiger entscheidet die Frau alleine über die Freizeitgestaltung bei Nicht-ALG-II-Beziehern. Das lässt die Vermutung zu, dass die Rolleneinstellungen in diesen prekären Lagen durch institutionelle Regelungen dominiert werden, die auf eine geschlechtsunabhängige Aktivierung zur Arbeitsaufnahme hinwirken.

In ALG-II-Haushalten mit Kindern unter 15 Jahren entscheidet seltener der Mann alleine über die Freizeitgestaltung. Dies scheint für Haushalte ohne ALG-II-Bezug nicht zuzutreffen. Der Ehestand hat in keiner der Gruppen einen statistisch signifikanten Einfluss auf die Machtverhältnisse. Ein schlechtes Familienklima geht bei Paaren ohne ALGII-Bezug häufig mit höherer individueller Entscheidungsmacht der beiden Partner bezüglich der Freizeitgestaltung einher. Gemeinsame Entscheidungen treten seltener auf, wenn häufiger Missverständnisse, Spannungen oder Konflikte im Haushalt vorliegen. Bei Paaren im Bezug hat lediglich der Mann häufiger die alleinige Entscheidungsmacht.

Die Ressourcen mit Bezug zu einer eventuellen Erwerbstätigkeit weisen ausschließlich in der Gruppe der Nicht-ALG-II-Bezieher statistisch signifikante Effekte auf. Hier zeigt sich für die Differenz im Nettoeinkommen zwischen den Partnern, dass Frauen bei einer ungleichmäßigen Verteilung der Nettoeinkommen seltener alleine über die Entscheidungsmacht verfügen. Sowohl bei einem höheren Nettoeinkommen des Mannes ist dies der Fall als auch dann, wenn die Frau mehr verdient. Dies ließe sich auf eine geschlechtsspezifisch unterschiedliche Bereitschaft zurückführen, den Partner über die Verwendung des eigenen Einkommens entscheiden zu lassen. Ein höherer gemeinsamer sozio-ökonomischer Status führt in Nicht-ALG-II-Partnerschaften dazu, dass die Frau seltener allein über die Freizeitgestaltung entscheidet. Mit einer längeren Arbeitszeit des Mannes geht eine höhere Entscheidungsmacht der Frau in dieser Gruppe einher. Dies deutet darauf hin, dass der Frau in diesem Fall mehr Zeit zur Verfügung steht, um die Freizeit zu planen und sie so eher die Möglichkeit hat zu entscheiden. 
Tabelle 3: Regressionsmodell - Entscheidung über Freizeitgestaltung

\begin{tabular}{|c|c|c|c|c|}
\hline & \multicolumn{2}{|c|}{ kein ALG-II-Bezug } & \multicolumn{2}{|c|}{ ALG-II-Bezug } \\
\hline & $\begin{array}{c}\text { Mann ent- } \\
\text { scheidet eher }\end{array}$ & $\begin{array}{c}\text { Frau ent- } \\
\text { scheidet eher }\end{array}$ & $\begin{array}{c}\text { Mann ent- } \\
\text { scheidet eher }\end{array}$ & $\begin{array}{c}\text { Frau ent- } \\
\text { scheidet eher }\end{array}$ \\
\hline \multicolumn{5}{|l|}{ individuelle Merkmale } \\
\hline weiblich & $-0,558^{* *}$ & 0,058 & $-0,686^{*}$ & $0,506^{*}$ \\
\hline Alter & $-0,020+$ & $-0,022^{\star *}$ & $-0,036+$ & 0,000 \\
\hline niedrige Bildung & 0,222 & $-0,076$ & $-0,808^{*}$ & $-0,562+$ \\
\hline mittlere Bildung & \multicolumn{2}{|c|}{ Referenzkategorie } & \multicolumn{2}{|l|}{ Referenzkategorie } \\
\hline hohe Bildung & $0,457+$ & $0,322+$ & 0,357 & 0,086 \\
\hline Migrationshintergrund & 0,115 & $-0,022$ & $-0,374$ & $-1,422+$ \\
\hline $\begin{array}{l}\text { Missverständnisse, Spannungen oder Konflikte im } \\
\text { Haushalt }\end{array}$ & $0,369^{* * *}$ & $0,435^{\star \star *}$ & $0,361+$ & $-0,054$ \\
\hline gemeinsames Konto & \multicolumn{2}{|l|}{ Referenzkategorie } & \multicolumn{2}{|l|}{ Referenzkategorie } \\
\hline eigenes Konto & 0,186 & $0,272+$ & 0,235 & 0,169 \\
\hline weder noch & $-0,714$ & 0,166 & 0,319 & 0,083 \\
\hline Einstellung der Zielperson zu Geschlechterrollen & $-0,236^{*}$ & $-0,055$ & $-0,127$ & $-0,117$ \\
\hline Religiosität & 0,026 & $0,183^{*}$ & 0,103 & 0,070 \\
\hline \multicolumn{5}{|l|}{ dyadische Merkmale } \\
\hline Mann 0 bis 5 Jahre älter & \multicolumn{2}{|l|}{ Referenzkategorie } & \multicolumn{2}{|l|}{ Referenzkategorie } \\
\hline Mann mehr als 5 Jahre älter & 0,094 & $-0,237$ & $-0,910^{*}$ & $-0,458$ \\
\hline Mann jünger & $-0,070$ & $-0,076$ & $-1,433^{*}$ & $-0,691+$ \\
\hline Mann höher gebildet & $-0,072$ & $-0,121$ & $-0,758$ & 0,420 \\
\hline gleiches Bildungsniveau & \multicolumn{2}{|c|}{ Referenzkategorie } & \multicolumn{2}{|c|}{ Referenzkategorie } \\
\hline Frau höher gebildet & $-0,180$ & $-0,104$ & $-0,030$ & $-0,186$ \\
\hline Wohnort in den neuen Bundesländern & $0,457+$ & 0,253 & $-0,606$ & 0,167 \\
\hline kein Migrationshintergrund & \multicolumn{2}{|l|}{ Referenzkategorie } & \multicolumn{2}{|l|}{ Referenzkategorie } \\
\hline nur Mann Migrationshintergrund & 0,293 & 0,235 & $-0,238$ & $1,521^{*}$ \\
\hline nur Frau Migrationshintergrund & 0,108 & 0,366 & $-0,447$ & 0,221 \\
\hline beide Migrationshintergrund & $-0,422$ & $-0,093$ & 0,506 & $2,078^{*}$ \\
\hline Ehepaar & 0,452 & 0,127 & $-0,484$ & $-0,646$ \\
\hline mindestens 1 Kind unter 15 Jahren im Haushalt & $-0,241$ & $-0,152$ & $-1,061^{*}$ & 0,283 \\
\hline Mann hat höheres Nettoeinkommen & $-0,041$ & $-0,868^{*}$ & 1,536 & $-1,338$ \\
\hline gleiche Nettoeinkommen & \multicolumn{2}{|l|}{ Referenzkategorie } & \multicolumn{2}{|l|}{ Referenzkategorie } \\
\hline Frau hat höheres Nettoeinkommen & $-0,144$ & $-0,950^{*}$ & 2,741 & 2,138 \\
\hline Summe Nettoeinkommen beider Partner & 0,000 & 0,000 & $-0,001$ & 0,001 \\
\hline Mann hat höheren sozio-ökonomischen Status & $-0,182$ & $-0,258$ & 1,813 & 0,037 \\
\hline gleicher Status & \multicolumn{2}{|c|}{ Referenzkategorie } & \multicolumn{2}{|l|}{ Referenzkategorie } \\
\hline Frau hat höheren sozio-ökonomischen Status & $-0,287$ & $-0,267$ & 1,331 & $-0,937$ \\
\hline Summe sozio-ökonomischer Status beider Partner & $-0,003$ & $-0,009^{*}$ & $-0,007$ & $-0,013$ \\
\hline Mann hat längere Arbeitszeit & $-0,069$ & $0,840^{*}$ & $-0,296$ & 0,992 \\
\hline gleiche Arbeitszeit & \multicolumn{2}{|l|}{ Referenzkategorie } & \multicolumn{2}{|l|}{ Referenzkategorie } \\
\hline Frau hat längere Arbeitszeit & 0,047 & 0,629 & $-1,649$ & $-0,865$ \\
\hline Summe Arbeitszeit beider Partner & 0,007 & 0,007 & $-0,023$ & $-0,006$ \\
\hline Arbeitslosengeld-II-Bezug & $-0,715$ & 0,777 & & \\
\hline Konstante & $-2,946^{* * *}$ & $-2,731^{\star * *}$ & $-3,655+$ & $-1,948$ \\
\hline
\end{tabular}

$\mathrm{N}=4.504 ; * * *: \mathrm{p}<0,001 ; * *: \mathrm{p}<0,01 ; *: \mathrm{p}<0,05 ;+\mathrm{p}<0,1$

Regressionskoeffizienten eines multinomialen logit-Modells mit random intercept auf der Paarebene;

Basiskategorie: „,beide in gleichem Maße/abwechselnd“

Quelle: Eigene Berechnungen (PASS SUF 2006-2010 V2, Welle 2) 
Der Mann entscheidet in ALG-II-Partnerschaften seltener allein, wenn die befragte Person über ein niedriges Bildungsniveau verfügt. Für die Frau lässt sich dies nicht beobachten. Hinsichtlich der Differenzen im Bildungsniveau beider Partner lassen sich keine Zusammenhänge mit dem Entscheidungsverhalten feststellen.

Wie auch im Modell zum Entscheidungsverhalten bei größeren Anschaffungen zeigt sich ein deutlicher Geschlechtereffekt im Antwortverhalten, der auf eine geschlechtsspezifisch unterschiedliche Einschätzung der Machtverhältnisse hindeutet. Für das Alter lässt sich in Bezug auf die Entscheidungen über die Freizeitgestaltung nur feststellen, dass in Partnerschaften ohne ALG-II-Bezug die Frau mit zunehmendem Alter der befragten Person seltener allein entscheidet. Die Altersdifferenz hat im Kontext des ArbeitslosengeldII-Bezuges einen statistisch signifikanten Einfluss: bei einer Altersdifferenz, unabhängig davon, in welche Richtung, entscheidet der Mann seltener als bei gleichaltrigen Paaren oder solchen, bei denen der Mann höchstens fünf Jahre älter ist. Auch im Modell zur Entscheidung über die Freizeitgestaltung zeigt sich in Bezug auf den Wohnort kein Ost/WestUnterschied. Die Migrationskonstellationen zeigen innerhalb der ALG-II-Bezieher zum Teil unterschiedliche Muster in der Verteilung der Entscheidungsmacht. Hat hier allein der Mann oder haben beide Partner einen Migrationshintergrund, trifft deutlich häufiger die Frau alleine die Entscheidung über die Freizeitgestaltung.

\section{Zusammenfassung und Diskussion}

Im vorliegenden Artikel wird davon ausgegangen, dass es sich bei größeren Anschaffungen häufig um unregelmäßige und wichtige Entscheidungen über die Verwendung von Geld in der Partnerschaft handelt (dirigierende Macht). Entscheidungen über die Gestaltung der gemeinsamen Freizeit umfassen neben Aspekten dirigierender Macht dagegen häufig auch kleinere, alltägliche Entscheidungen, die lediglich geringe oder keine Kosten verursachen und vielmehr zur ausführenden Macht gezählt werden können (SafiliosRothschild 1976: 359). Betrachtet man die Befunde zur Verteilung der Macht in diesen beiden Entscheidungskontexten im Zusammenhang, ergibt sich für die Machtverteilung ein vielfältiges Bild.

Die theoretisch hergeleiteten Vermutungen über den Einfluss von Ressourcen und Geschlechterrolleneinstellungen müssen insgesamt differenziert betrachtet werden. Der Einfluss von Geschlechterrolleneinstellungen lässt sich deutlich belegen. Insbesondere für die größeren Anschaffungen zeigt sich, dass eine modernere Rolleneinstellung häufig mit egalitärem Entscheidungsverhalten einhergeht. Für die Freizeitgestaltung lässt sich dies nur zum Teil bestätigen. In der ALG-II-Gruppe haben Rolleneinstellungen hier keinen Einfluss, was unter Umständen darauf zurückzuführen ist, dass unter der Vorgabe des „Förderns und Forderns" beide Partner geschlechtsunabhängig zur Arbeitsaufnahme angehalten werden und so die realen Geschlechterarrangements die u.U. divergierenden Rolleneinstellungen dominieren.

Die ressourcentheoretischen Überlegungen zur Machtverteilung, die sich auf die Erwerbstätigkeit der beiden Partner beziehen, lassen sich nur sehr bedingt in den vorliegenden Analysen nachweisen. Lediglich im Rahmen der Freizeitgestaltung zeigen sich überhaupt Effekte der Differenz in den Ressourcenausstattungen und dies auch nur für Per- 
sonen ohne ALG-II-Bezug. Eine Interpretation der Befunde wäre, dass im ALG-IIKontext die unterschiedliche Ressourcenausstattung der Partner tatsächlich keinen Einfluss mehr auf das Entscheidungsverhalten hat. Dies erscheint nicht unplausibel, da die vorhandenen Differenzen nicht besonders groß sind und damit zu unbedeutend sein könnten, um Einfluss auf die Entscheidungsprozesse in der Partnerschaft zu nehmen.

Andererseits befindet sich unter den ALG-II-Beziehern im Sample eine große Anzahl von arbeitslosen Paaren. Da diese Paare weder beim Nettoeinkommen, noch beim sozioökonomischen Status oder der Arbeitszeit Werte größer Null aufweisen, gibt es wenig Varianz auf diesen Variablen, die es zulassen würde, stabile Effekte zu schätzen. Dieser Eindruck verstärkt sich, wenn man berücksichtigt, dass die Koeffizienten zum Teil sehr groß, gleichzeitig aber nicht statistisch signifikant sind. Eine weitere Ursache könnte die hohe Korrelation zwischen dem Nettoeinkommen, dem sozio-ökonomischen Status und der Arbeitszeit sein. Dies könnte dazu führen, dass die zugehörigen Koeffizienten unter Kontrolle der jeweils anderen Merkmale nicht mehr statistisch signifikant werden. ${ }^{10}$

Ein etwas deutlicheres Bild zeigt sich hinsichtlich der Ressource Bildung. In der ALG-II-Gruppe zeigt sich sowohl für die Entscheidungen über größere Anschaffungen als auch über die Freizeitgestaltung ein deutlicher Einfluss des Bildungsniveaus der befragten Person. In beiden Modellen geben Befragte mit einer niedrigen Bildung an, dass eher egalitär entschieden wird. Geht man davon aus, dass höher gebildete Personen ein geringeres Risiko haben, auf Arbeitslosengeld II angewiesen zu sein, kann man vermuten, dass diese die Abhängigkeit von staatlichen Transferleistungen als besonders belastend empfinden. Diese Belastungen könnten dann wiederum Einfluss auf die Partnerschaft haben, in diesem Fall auf die Fähigkeit gemeinschaftlich Entscheidungen zu treffen.

Neben den Befunden zu Rolleneinstellungen und den eher eingeschränkten Interpretationsmöglichkeiten der Ressourcenausstattung in Bezug auf das Entscheidungsverhalten zeigen die Analysen noch weitere Differenzen in den Erklärungsmustern für die Entscheidungsmacht innerhalb von Paaren auf. Insbesondere fällt auf, dass für Paare im ALG II Spannungen und Konflikte weniger Einfluss auf die Machtverteilung in den Entscheidungsprozessen nehmen. Auch hier würden wir vermuten, dass der institutionelle Kontext des ALG II eine Rolle spielt.

Befindet sich ein Kind unter 15 Jahren im Haushalt, hat dies in Abhängigkeit vom ALG-II-Bezug einen Einfluss auf das Entscheidungsverhalten der Paare. Sind es bei den Nicht-ALG-II-Haushalten die Entscheidungen über größere Anschaffungen, auf die die Anwesenheit eines Kindes Einfluss nimmt, sind es in der ALG-II-Gruppe die Entscheidungen über die Freizeitgestaltung.

Die Art der Kontoführung hat in Bezug auf die Entscheidung über größere Anschaffungen einen deutlichen Einfluss: Haben die Partner getrennte Konten, so entscheiden sie auch häufiger getrennt. Dies gilt weitgehend unabhängig davon, ob es sich um Paare mit oder ohne ALG-II-Bezug handelt. Bei den Entscheidungen über die Freizeitgestaltung spielt die Kontoführung dagegen keine Rolle. Dies erscheint intuitiv plausibel, da es sich

10 Leider lässt sich dieses Problem im Rahmen von nicht-linearen Modellen, wie dem vorliegenden multinomialen Logit-Modell, nicht ohne weiteres durch stufenweise Aufnahme der Regressoren lösen wie es bei linearen Modellen möglich ist (vgl. Mood 2010). In gängigen Statistikpaketen implementierte Algorithmen, die dieses Problem auch für die hier verwendeten Mehrebenenmodelle mit Interaktionstermen lösen würden, liegen nach dem Wissen der Autoren zur Zeit nicht vor. 
bei größeren Anschaffungen meist um Entscheidungen mit monetärem Bezug handelt, während die Gestaltung der gemeinsamen Freizeit auch häufig Entscheidungen umfasst, die keine Kosten verursachen.

Die vorliegenden Analysen beleuchten einen Gegenstandsbereich, zu dem bisher keinerlei Befunde vorliegen. Gleichzeitig muss aber auch festgestellt werden, dass ein weiterer Ausbau der Untersuchung des Entscheidungsverhaltens von Paaren im ALG-II-Bezug und ähnlich prekären Lagen notwendig ist. Dies gilt sowohl in methodischer als auch theoretischer Hinsicht. Methodisch wäre es wünschenswert, mit zusätzlichen Daten die oben angeführten Probleme in der Modellschätzung zu reduzieren, sowie den Einfluss des ALG-II-Bezugs in längsschnittlichen Analysen näher zu untersuchen. Daneben empfiehlt sich auch auf Basis der nach und nach zunehmend umfangreicheren Befundlage zur Situation von Arbeitslosengeld-II-Beziehern das theoretische Gerüst zur Lage von Paaren im Leistungsbezug zu erweitern. Hier wäre dann auch eine weitergehende geschlechtertheoretische Differenzierung von männlichen und weiblichen Antwortmustern interessant.

\section{Literatur}

Addo, F. R. \& Sassler, S. (2010). Financial arrangements and relationship quality in low-income couples. Family Relations, 59, S. 408-423.

Andreoni, J., Brown, E. \& Rischall, I. (2003). Charitable giving by married couples. Who decides and why does it matter? The Journal of Human Resources, 38, 1, S. 111-133.

Berg, M. et al. (2011). Codebuch und Dokumentation des „Panel Arbeitsmarkt und soziale Sicherung“ (PASS). Band I: Datenreport Welle 4. Nürnberg: Institut für Arbeitsmarkt- und Berufsforschung (FDZ Datenreport 08/2011).

Blood, R. O. \& Wolfe. D. M. (1978 [1960]). Husbands and wives. The dynamics of married living. Westport: Greenwood Press.

Blumstein, P. \& Schwartz. P. (1983). American couples: Money, work, sex. New York: Morrow.

Brauns, H. \& Steinmann. S, (1999). Educational reform in France, West-Germany, and the United Kingdom. Updating the CASMIN educational classification. ZUMA-Nachrichten, 23, 44, S. 7-44.

Brehmer, W., Klenner, C. \& Klammer, U. (2010). Wenn Frauen das Geld verdienen - eine empirische Annäherung an das Phänomen der „Familienernährerin“. Düsseldorf: Wirtschafts- und Sozialwissenschaftliches Institut in der Hans-Böckler-Stiftung (WSI-Diskussionspapier 170).

Centers, R., Raven. B. H. \& Rodrigues, A. (1971). Conjugal power structure: A re-examination“. American Sociological Review, 36, 2, S. 264-278.

Cosenza, R. M. \& Davis, D. L. (1981). Family vacation decision making over the family life cycle: A decision and influence structure analysis. Journal of Travel Research, 20, S. 17-23.

Fodness, D. (1992). The impact of family life cycle on the vacation decision-making process. Journal of Travel Research, 31, 2, S. 8-13.

Ganzeboom, H. B. G. \& Treiman, D. (2003). Three internationally standardised measures for comparative research on occupational status. In: Hoffmeyer-Zlotnik, J. H. P. \& Wolf, C. (Hrsg.), Advances in cross-national comparison. A European working book for demographic and socio-economic variables. New York u. a.: Kluwer Academic/Plenum Publishers, S. 159-193.

Goffman, E. (2001). Interaktion und Geschlecht. Frankfurt am Main: Campus (2. Auflage).

Grün, C., Hauser, W. \& Rhein, T. (2008). Finding a job: Consequences for life satisfaction and interactions with job quality. Nürnberg: Institut für Arbeitsmarkt- und Berufsforschung (IAB-Discussion Paper 24/2008).

Götz, S., Ludwig-Mayerhofer, W. \& Schreyer, F. (2010). Sanktionen im SGB II: Unter dem Existenzminimum. Nürnberg: Institut für Arbeitsmarkt- und Berufsforschung (IAB-Kurzbericht 10/2010). 
Heer, D. M. (1963). The measurement and bases of family power: An overview. Marriage and Family Living, 25, 2, S. 13-139.

Hess, D., Hartenstein, W. \& Smid, M. (1991). Auswirkungen von Arbeitslosigkeit auf die Familie. Nürnberg: Institut für Arbeitsmarkt- und Berufsforschung (Mitteilungen aus der Arbeitsmarkt- und Berufsforschung 1/2010).

Hillmert, S. (2002). Familiale Ressourcen und Bildungschancen: Konsequenzen eines frühzeitigen Elternverlustes. Zeitschrift für Familienforschung, 14, 1, S. 44-69.

Hirseland, A. \& Ramos Lobato, P. (2010). Armutsdynamik und Arbeitsmarkt. Entstehung, Verfestigung und Überwindung von Hilfebedürftigkeit bei Erwerbsfähigen. Nürnberg: Institut für Arbeitsmarktund Berufsforschung (IAB-Forschungsbericht 3/2010).

Jaehrling, K. (2010). Alte Routinen, neue Stützen - Zur Stabilisierung von Geschlechterasymmetrien im SGB II. In: Jaehrling, K. \& Rudolph, C. (Hrsg.), Grundsicherung und Geschlecht. Gleichstellungspolitische Befunde zu den Wirkungen von ,Hartz IV“. Münster: Westfälisches Dampfboot.

Jahoda, M., Lazarsfeld, P. F. \& Zeisel, H. (2007 [1933]). Die Arbeitslosen von Marienthal: Ein soziographischer Versuch über die Wirkungen langandauernder Arbeitslosigkeit. Frankfurt am Main: Suhrkamp.

Kalmijn, M. \& Bernasco, W. (2000). The ties that bind: A study of individualism in married and cohabiting couples in the Netherlands. In: Weesie, J. \& Raub, W. (Hrsg.), The management of durable relations. Theoretical models and empirical studies of households and organizations. Amsterdam: Thela Thesis Publishers.

Kenny, D., Kashy, D. A. \& Cook, W. L. (2006). Dyadic data analysis. New York, London: Guilford Press.

Kirchler, E. (1988). Diary reports on daily economic decisions of happy versus unhappy couples. Journal of Economic Psychology, 9, S. 327-357.

Kirchler, E. (1995). Studying economic decisions within private households: A critical review and design for a „,couple experiences diary”. Journal of Economic Psychology, 16, S. 393-419.

Kirchler, E., Hoelzl, E. \& Kamleitner, B. (2008). Spending and credit use in the private household. The Journal of Socio-Economics, 37, S. 519-532.

Kollock, P., Blumstein, P. \& Schwartz, P. (1994). The judgment of equity in intimate relationships. Social Psychology Quarterly, 57, 4, S. 340-351.

Kranichfeld, M. L. (1987). Rethinking family power. Journal of Family Issues, 8, S. 42-56.

Lenz, K. (2009). Soziologie der Zweierbeziehung. Eine Einführung. Wiesbaden: VS Verlag für Sozialwissenschaften (4. Auflage).

Lise, J. \& Seitz, S. (2011). Consumption inequality and intra-household allocations. Review of Economic Studies, 78, S. 328-355.

Lorber, J. (1999). Gender-Paradoxien. Opladen: Leske + Budrich.

Lorber, J. \& Farrell, S. A. (Hrsg.) (1991). The social construction of gender. Newbury Park, London, New Delhi: Sage Publications.

Lott, Y. (2009). Verwaltung und Entscheidung - Bestimmt das individuelle Einkommen die Machtverteilung in Paarbeziehungen? Kölner Zeitschrift für Soziologie und Sozialpsychologie, 61, 3, S. 327-353.

Ludwig-Mayerhofer, W. (2000). Transaction costs, power, and gender attitudes in financial arrangements of couples. In: Wessie. J. \& Raub, W. (Hrsg.), The management of durable relations: Theoretical models and empirical studies of households and organizations. Amsterdam: Thela Thesis Publishers.

Ludwig-Mayerhofer, W. (2006). Geldverwaltung und -verteilung in Paarbeziehungen. Zeitschrift für Sozialreform, 52, 4, S. 467-491.

Ludwig-Mayerhofer, W., Gartner, H. \& Allmendinger, J. (2006). The allocation of money in couples: The end of inequality? Zeitschrift für Soziologie, 35, 3, S. 212-226.

Mohr, G. \& Richter, P. (2008). Arbeitslosigkeit: Psychosoziale Folgen. Aus Politik und Zeitgeschichte, $40-41$, S. 25-32.

Mood, C. (2010). Logistic regression: Why we cannot do what we think we can do, and what we can do about it. European Sociological Review, 26, S. 67-82. 
Mottiar, Z. \& Quinn, D. (2004). Couple dynamics in household tourism decision making: Women as the gatekeepers? Journal of Vacation Marketing, 10, 2, S. 149-160.

Nave-Herz, R. (2004). Ehe- und Familiensoziologie. Eine Einführung in Geschichte, theoretische Ansätze und empirische Befunde. Weinheim, München: Juventa Verlag.

Nichols, C. M. \& Snepenger, D. J. (1988). Family decision making and tourism. Behavior and attitudes. Journal of Travel Research, 26, 4, S. 2-6.

Nye, I. F. (1978). Is choice and exchange theory the key? Journal of Marriage and the Family, 40, 2, S. 219-233.

Nye, I. F. (1980). Family mini theories as special instances of choice and exchange theory. Journal of Marriage and the Family, 42, 3, S. 479-489.

Pahl, J. (1980). Patterns of money management within marriage. Journal of Social Policy, 9, S. 313-335.

Pahl, J. (1983). The allocation of money and the structuring of inequality within marriage. Sociological Review, 31, 2, S. 237-262.

Pahl, J. (1990). Household spending, personal spending and the control of money in marriage. Sociology, 24, 1, S. 119-138.

Pahl, J. (1995). His money, her money: Recent research on financial organisation in marriage. Journal of Economic Psychology, 16, S. 361-376.

Rabe-Hesketh, S. \& Skrondal, A. (2004). GLLAMM manual. Berkeley: University of California (U.C. Berkeley Division of Biostatistics Working Paper Series 160).

Rosenthal, C. J. (1985). Kinkeeping in the familial division of labor. Journal of Marriage and the Family, 47, 4, S. 965-974.

Rudolph, C. (2010). Vergeschlechtlichungsprozesse im SGB II und gleichstellungspolitische Interventionen. In: Jaehrling, K. \& Rudolph, C. (Hrsg.), Grundsicherung und Geschlecht. Gleichstellungspolitische Befunde zu den Wirkungen von ,Hartz IV“. Münster: Westfälisches Dampfboot.

Safilios-Rothschild, C. (1970). The study of family power structure: A review 1960-1969. Journal of Marriage and the Family, 32, 4, S. 539-552.

Safilios-Rothschild, C. (1976). A macro- and micro-examination of family power and love: An exchange model. Journal of Marriage and the Family, 38, 2, S. 355-362.

Skrondal, A. \& und Rabe-Hesketh, S. (2004). Generalized latent variable modelling. Multilevel, longitudinal, and structural equation models. Boca Raton u. a.: Chapman \& Hall/CRC.

Stamp, P. (1985). Research note: Balance of financial power in marriage: An exploratory study of breadwinning wives. Sociological Review, 33, S. 546-557.

Tichenor, V. J. (1999). Status and income as gendered resources: The case of marital power. Journal of Marriage and the Family, 61, 3, S. 638-650.

van Raaji, W. F. \& Francken, D. A. (1984). Vacation decisions, activities, and satisfactions. Annals of Tourism Research, 11, S. 101-112.

Vogler, C. (1998). Money in the household: Some underlying issues of power. The Sociological Review, 46, 4, S. 687-713.

Vogler, C. (2005). Cohabiting couples: Rethinking money in the household at the beginning of the twenty first century. The Sociological Review. 53, 1, S. 1-29.

Vogler, C., Lyonette, C. \& Wiggins, R. D. (2008). Money, power and spending decisions in intimate relationships. The Sociological Review, 56. 1, S. 117-143.

Vogler, C. \& Pahl, J. (1993). Social and economic change and the organisation of money within marriage. Work, Employment \& Society, 7, 1, S. 71-95.

Weber, M. (2005). Wirtschaft und Gesellschaft. Neu-Isenburg: Melzer Verlag.

Wenzel, U. (2008). Fördern und Fordern aus Sicht der Betroffenen: Verstehen und Aneignung sozialund arbeitsmarktpolitischer Maßnahmen des SGB II. Zeitschrift für Sozialreform, 54, 1, S. 57-78.

West, C. \& Fenstermaker, S. (1995). Doing difference. In: Gender and Society, 9, 1, S. 8-37.

West, C. \& Zimmerman, D. H. (1987). Doing gender. In: Gender and Society, 1, 2, S. 125-151.

West, C. \& Zimmerman, D. H. (2002). Doing gender. In: Fenstermaker, S. \& West, C. (Hrsg.), Doing gender, doing difference. Inequality, power, and institutional change. New York: Routledge, S. 323. 
Wiepking, P. \& Bekkers, R. (2010). Does who decides really matter? Causes and consequences of personal financial management in the case of larger and structural charitable donations. Voluntas, 21, S. 240-263.

Eingereicht am/Submitted on: 24.10.2011

Angenommen am/Accepted on: 18.07.2012

Anschriften des Autors und der Autorin/Addresses of the authors:

Arne Bethmann, Diplom-Sozialwirt (Korrespondenzautor/corresponding author)

Institut für Arbeitsmarkt- und Berufsforschung (IAB)

Regensburger Straße 104

90478 Nürnberg

Deutschland/Germany

Anne Berngruber, Diplom-Sozialwirtin

Deutsches Jugendinstitut (DJI)

Nockherstraße 2

81541 München

Deutschland/Germany

E-Mail: Arne.Bethmann@iab.de berngruber@dji.de 


\section{Anhänge}

Tabelle 4: Beschreibung der Merkmale des Analysesamples (Individualebene nach Geschlecht der Zielpersonen, kategoriale Merkmale)

\begin{tabular}{|c|c|c|c|c|c|c|}
\hline & \multicolumn{2}{|c|}{ Männer } & \multicolumn{2}{|c|}{ Frauen } & \multicolumn{2}{|c|}{ Gesamt } \\
\hline & $\%$ & $N$ & $\%$ & $n$ & $\%$ & $N$ \\
\hline \multicolumn{7}{|c|}{ Entscheidungen über größere Anschaffungen } \\
\hline Mann entscheidet eher & 14,51 & 347 & 10,29 & 227 & 12,55 & 574 \\
\hline beide entscheiden gemeinsam & 77,71 & 1.915 & 81,07 & 1.957 & 79,27 & 3.872 \\
\hline Frau entscheidet eher & 7,99 & 212 & 8,64 & 294 & 8,18 & 506 \\
\hline Gesamt & 100,00 & 2.474 & 100,00 & 2.478 & 100,00 & 4.952 \\
\hline \multicolumn{7}{|c|}{ Entscheidungen über die Freizeitgestaltung } \\
\hline Mann entscheidet eher & 7,20 & 161 & 4,13 & 104 & 5,78 & 265 \\
\hline beide entscheiden gemeinsam & 81,23 & 2.029 & 82,39 & 2.033 & 81,77 & 4.062 \\
\hline Frau entscheidet eher & 11,57 & 285 & 13,48 & 337 & 12,45 & 622 \\
\hline Gesamt & 100,00 & 2.475 & 100,00 & 2.474 & 100,00 & 4.926 \\
\hline \multicolumn{7}{|l|}{ Bildung (CASMIN, 3-stufig) } \\
\hline niedrig & 45,35 & 1.049 & 43,53 & 940 & 44,51 & 1.989 \\
\hline mittel & 29,86 & 901 & 38,94 & 1.179 & 34,06 & 2.080 \\
\hline hoch & 24,79 & 581 & 17,52 & 403 & 21,42 & 984 \\
\hline Gesamt & 100,00 & 2.531 & 100,00 & 2.522 & 100,00 & 5.053 \\
\hline \multicolumn{7}{|l|}{ Migrationshintergrund } \\
\hline kein & 82,19 & 2.020 & 81,54 & 2.012 & 81,89 & 4.032 \\
\hline 1. oder 2. Generation & 17,81 & 492 & 18,46 & 503 & 18,11 & 995 \\
\hline Gesamt & 100,00 & 2.512 & 100,00 & 2.515 & 100,00 & 5.027 \\
\hline \multicolumn{7}{|l|}{ Bankkonto } \\
\hline gemeinsames & 66,07 & 1.589 & 60,65 & 1.501 & 63,56 & 3.090 \\
\hline eigenes & 32,97 & 843 & 38,52 & 930 & 35,54 & 1.773 \\
\hline weder noch & 0,96 & 46 & 0,83 & 47 & 0,90 & 93 \\
\hline Gesamt & 100,00 & 2.478 & 100,00 & 2.478 & 100,00 & 4.956 \\
\hline
\end{tabular}

$\%=$ Anteilswert in Prozent (gewichtet); $n=$ Absolutwert (ungewichtet)

Quelle: Eigene Berechnungen (PASS SUF 2006-2010 V2, Welle 2)

Tabelle 5: Beschreibung der Merkmale des Analysesamples (Individualebene nach Geschlecht der Zielpersonen, metrische Merkmale)

\begin{tabular}{|c|c|c|c|c|c|c|}
\hline & \multicolumn{2}{|c|}{ Männer } & \multicolumn{2}{|c|}{ Frauen } & \multicolumn{2}{|c|}{ Gesamt } \\
\hline & $\varnothing$ & $S D$ & $\varnothing$ & $S D$ & $\varnothing$ & $S D$ \\
\hline \multicolumn{7}{|l|}{ Alter } \\
\hline in Jahren & 50,4 & 12,8 & 48,1 & 14,0 & 49,3 & 13,4 \\
\hline \multicolumn{7}{|l|}{ Einstellung zu Geschlechterrollen } \\
\hline vierstufig, 1 „traditionell“ bis 4 „modern“ & 2,84 & 0,85 & 2,92 & 0,90 & 2,88 & 0,88 \\
\hline \multicolumn{7}{|l|}{ Missverständnisse, Spannungen oder Konflikte im Haushalt } \\
\hline fünfstufig, 1 „sehr selten oder nie“ bis 5 „,sehr häufig“ & 2,42 & 0,73 & 2,48 & 0,83 & 2,45 & 0,78 \\
\hline \multicolumn{7}{|l|}{ Religiosität } \\
\hline vierstufig, 1 „überhaupt nicht religiös“ bis 4 „,sehr religiös“ & 2,32 & 0,73 & 2,53 & 0,78 & 2,42 & 0,76 \\
\hline
\end{tabular}

$\varnothing=$ arithmetischer Mittelwert (gewichtet); $S D=$ Standardabweichung (gewichtet)

Quelle: Eigene Berechnungen (PASS SUF 2006-2010 V2, Welle 2) 
Tabelle 6: Beschreibung des Analysesamples (dyadische Paarebene nach Arbeitslosengeld-II-Bezug, kategoriale Merkmale)

\begin{tabular}{|c|c|c|c|c|c|c|}
\hline & \multicolumn{4}{|c|}{ Arbeitslosengeld-II-Bezug } & & \\
\hline & \multicolumn{2}{|c|}{ ohne } & \multicolumn{2}{|c|}{ mit } & \multicolumn{2}{|c|}{ Gesamt } \\
\hline & $\%$ & $n$ & $\%$ & $n$ & $\%$ & $n$ \\
\hline \multicolumn{7}{|l|}{ Altersdifferenz } \\
\hline Mann 0 bis 5 Jahre älter & 62,91 & 1.199 & 55,36 & 289 & 62,56 & 1.488 \\
\hline Mann mehr als 5 Jahre älter & 22,28 & 434 & 26,31 & 171 & 22,46 & 605 \\
\hline Mann jünger & 14,81 & 310 & 18,34 & 121 & 14,98 & 431 \\
\hline Gesamt & 100,00 & 1.943 & 100,00 & 581 & 100,00 & 2.524 \\
\hline \multicolumn{7}{|l|}{ Bildungsdifferenz } \\
\hline Mann höher gebildet & 25,55 & 449 & 19,65 & 99 & 25,28 & 548 \\
\hline gleiches Bildungsniveau & 56,95 & 1.081 & 62,94 & 351 & 57,23 & 1.432 \\
\hline Frau höher gebildet & 17,50 & 401 & 17,41 & 123 & 17,49 & 524 \\
\hline Gesamt & 100,00 & 1.931 & 100,00 & 573 & 100,00 & 2.504 \\
\hline \multicolumn{7}{|l|}{ Familienstand } \\
\hline Ehepaar & 94,61 & 1.695 & 84,23 & 449 & 94,13 & 2.144 \\
\hline nicht-eheliche Lebensgemeinschaft & 5,39 & 248 & 15,77 & 132 & 5,87 & 380 \\
\hline Gesamt & 100,00 & 1.943 & 100,00 & 581 & 100,00 & 2.524 \\
\hline \multicolumn{7}{|l|}{ Kinder im Haushalt } \\
\hline kein Kind unter 15 Jahren & 68,66 & 1.232 & 35,46 & 296 & 67,13 & 1.528 \\
\hline mind. 1 Kind unter 15 Jahren & 31,34 & 711 & 64,54 & 285 & 32,87 & 996 \\
\hline Gesamt & 100,00 & 1.943 & 100,00 & 581 & 100,00 & 2.524 \\
\hline \multicolumn{7}{|l|}{ Region des Wohnorts } \\
\hline alte Bundesländer & 80,91 & 1.547 & 65,58 & 340 & 80,21 & 1.887 \\
\hline neue Bundesländer & 19,09 & 396 & 34,42 & 241 & 19,79 & 637 \\
\hline Gesamt & 100,00 & 1.943 & 100,00 & 581 & 100,00 & 2.524 \\
\hline \multicolumn{7}{|l|}{ Migrationskonstellation } \\
\hline kein Migrationshintergrund & 77,11 & 1.507 & 50,05 & 326 & 75,86 & 1.833 \\
\hline nur Mann Migrationshintergrund & 5,77 & 118 & 3,34 & 32 & 5,66 & 150 \\
\hline nur Frau Migrationshintergrund & 8,82 & 125 & 3,38 & 35 & 8,57 & 160 \\
\hline beide Migrationshintergrund & 8,29 & 160 & 43,22 & 174 & 9,91 & 334 \\
\hline Gesamt & 100,00 & 1.910 & 100,00 & 567 & 100,00 & 2.477 \\
\hline \multicolumn{7}{|l|}{ Einkommensdifferenz } \\
\hline Mann hat höheres Nettoeinkommen & 53,07 & 1.073 & 16,71 & 104 & 51,34 & 1.177 \\
\hline gleiche Nettoeinkommen & 32,33 & 545 & 64,21 & 406 & 33,84 & 951 \\
\hline Frau hat höheres Nettoeinkommen & 14,60 & 257 & 19,08 & 62 & 14,82 & 319 \\
\hline Gesamt & 100,00 & 1.875 & 100,00 & 572 & 100,00 & 2.447 \\
\hline \multicolumn{7}{|c|}{ Differenz im sozio-ökonomischen Status } \\
\hline Mann hat höheren Status & 43,50 & 900 & 18,81 & 121 & 42,36 & 1.021 \\
\hline gleicher Status & 33,05 & 576 & 60,86 & 382 & 34,33 & 958 \\
\hline Frau hat höheren Status & 23,45 & 462 & 20,33 & 70 & 23,31 & 532 \\
\hline Gesamt & 100,00 & 1.938 & 100,00 & 573 & 100,00 & 2.511 \\
\hline \multicolumn{7}{|l|}{ Arbeitszeitdifferenz } \\
\hline Mann hat längere Arbeitszeit & 54,04 & 1.119 & 18,86 & 118 & 52,40 & 1.237 \\
\hline gleiche Arbeitszeit & 31,52 & 538 & 60,87 & 382 & 32,88 & 920 \\
\hline Frau hat längere Arbeitszeit & 14,45 & 261 & 20,27 & 69 & 14,72 & 330 \\
\hline Gesamt & 100,00 & 1.918 & 100,00 & 569 & 100,00 & 2.487 \\
\hline
\end{tabular}

$\%=$ Anteilswert in Prozent (gewichtet); $n=$ Absolutwert (ungewichtet)

Quelle: Eigene Berechnungen (PASS SUF 2006-2010 V2, Welle 2) 
Tabelle 7: Beschreibung des Analysesamples (dyadische Paarebene nach Arbeitslosengeld-II-Bezug, metrische Merkmale)

\begin{tabular}{|c|c|c|c|c|c|c|}
\hline & \multicolumn{4}{|c|}{ Arbeitslosengeld-II-Bezug } & & \\
\hline & \multicolumn{2}{|c|}{ ohne } & \multicolumn{2}{|c|}{ mit } & \multicolumn{2}{|c|}{ Gesamt } \\
\hline & $\varnothing$ & $S D$ & $\varnothing$ & $S D$ & $\varnothing$ & $S D$ \\
\hline \multicolumn{7}{|c|}{ Summe Nettoeinkommen beider Partner } \\
\hline in Euro & 1871 & 1425 & 311 & 936 & 1795 & 1586 \\
\hline \multicolumn{7}{|c|}{ Summe sozio-ökonomischer Status beider Partner } \\
\hline in Punkten auf der ISEI-Skala & 49,3 & 34,4 & 12,1 & 36,5 & 47,5 & 38,2 \\
\hline \multicolumn{7}{|c|}{ Summe Arbeitszeit beider Partner } \\
\hline in Stunden & 44,2 & 27,7 & 13,8 & 39,2 & 42,7 & 30,9 \\
\hline
\end{tabular}

$\varnothing=$ arithmetischer Mittelwert (gewichtet); $S D=$ Standardabweichung (gewichtet)

Quelle: Eigene Berechnungen (PASS SUF 2006-2010 V2, Welle 2) 